\title{
Current Practice Patterns in Anterior Cruciate Ligament Reconstruction Among Fellowship-Trained Military Orthopaedic Surgeons
}

\author{
Thomas B. Lynch, M.D., Jeanne C. Patzkowski, M.D., Erin R. Swan, M.D., \\ Christopher J. Roach, M.D., Matthew R. Schmitz, M.D., \\ Jonathan F. Dickens, M.D., MOTION Group, and Andrew J. Sheean, M.D.
}

\begin{abstract}
Purpose: To evaluate current practice patterns in anterior cruciate ligament reconstruction (ACLR) surgery among fellowship-trained military surgeons. Methods: The MOTION database is a prospectively collected dataset of intraoperative variables across the Military Health System. This database was queried using Current Procedural Terminology code 29888 for ACLR among active-duty service members between October 2016 and December 2019. The intraoperative data pertaining to ACLR involving both isolated primary ACLRs and primary ACLRs combined with meniscal or chondral injuries were extracted with patient age, sex, and rank. Results: Two hundred sixty-six primary ACLRs performed by 21 fellowship-trained orthopaedic surgeons at 9 MTFs were identified. The mean age of patients undergoing ACLR was $27.2 \pm 7.7$ years. Bone-patellar tendon-bone autograft was the most commonly used graft source (137 of 266 [51.5\%] cases.) Meniscal injuries were treated with an isolated debridement in 53 of $156(34.0 \%)$ tears, whereas meniscal repair was performed in 86 of $156(55.1 \%)$ tears. Concomitant chondral pathology was noted in 43 of 266 cases $(16.2 \%)$ and most commonly addressed with chondroplasty (25 of 49 [51.0\%] chondral lesions). Conclusions: Bone-patellar tendon-bone autograft was the most commonly used graft type in ACLR among fellowship-trained surgeons treating active-duty service members. Concomitant meniscal pathology was encountered at rates comparable with what has been previously reported, and meniscal repair was favored over meniscal debridement in more than $50 \%$ cases. Level of Evidence: Level IV: Therapeutic case series.
\end{abstract}

$\mathbf{P}$ atient registries can be powerful clinical tools that facilitate the prospective collection of treatmentrelated and patient-reported outcomes data. The Multicenter Orthopaedic Outcomes Network (MOON) and Multicenter Anterior Cruciate Ligament (ACL) Revision Study (MARS) projects are 2 of the established

From San Antonio Military Medical Center, Fort Sam Houston, Texas, U.S.A. (T.B.L., J.C.P., E.R.S., C.J.R., M.R.S., A.J.S.); Walter Reed National Military Medical Center, Bethesda, Maryland, U.S.A. (J.F.D.).

The authors report the following potential conflicts of interest or sources of funding: A.J.S. reports personal fees from Arthroscopy, grants from Embody Parts, Inc, outside the submitted work. Full ICMJE author disclosure forms are available for this article online, as supplementary material.

Received February 25, 2020; accepted June 12, 2020.

Address correspondence to: Thomas B. Lynch, M.D., San Antonio Military Medical Center, 3551 Roger Brooke Dr., Fort Sam Houston, TX 78234.

E-mail: tblynch@gwu.edu

Published by Elsevier on behalf of the Arthroscopy Association of North America. This is an open access article under the CC BY-NC-ND license (http://creativecommons.org/licenses/by-nc-nd/4.0/).

2666-061X/20213

https://doi.org/10.1016/j.asmr.2020.06.003 data-collection platforms in use across multiple centers to prospectively collect clinical data pertaining to anterior cruciate ligament reconstruction (ACLR) and revision ACLR. ${ }^{1}$ These multicenter outcomes tracking networks have provided researchers and clinicians with a tremendous amount of information that has changed current practices in the surgical management of ACL injuries.

To date, the majority of military studies involving prospectively collected patient-reported outcome data have been accomplished in relative isolation without a reliable means for sharing and/or collating data collected across multiple military treatment facilities (MTFs). In response to this conspicuous capability gap, the Military Orthopaedic Tracking Injuries and Outcomes Network (MOTION) platform was developed as a web-based patient registry to assess the military-specific variables of injury and return to duty as well as the treatment and patient-reported outcomes. This triservice (United States Army, Air Force, and Navy) musculoskeletal data network allows military orthopaedic surgeons across MTFs to input demographic 
information, prospectively collect patient-reported outcomes, and catalog intraoperative details related to specific surgical procedures. Currently, there are 9 MTFs across the Department of Defense using the MOTION platform to prospectively collect clinical data relevant to a myriad of musculoskeletal conditions, the most common of which being ligamentous knee injuries and glenohumeral instability.

Multiple authors have reported on the results of surgeon surveys related to the surgical management of ACL injuries. ${ }^{1-4}$ In fact, since 1986, 34 such reports have been published based on the results of surveys administered to physicians. ${ }^{1}$ These survey results can have important effects on informing a broader understanding of practice patterns, specifically with respect to preferred graft type, graft-fixation methods, femoral tunnel drilling techniques, and the manner by which associated meniscal and/or chondral pathology is managed. However, studies of this type based on survey results can be limited by low surgeon response rates, surgeons' recall bias, and/or imprecise generalizations of one's own surgical practices. In addition, we are aware of only one such survey-based report related to ACLR practices among military surgeons, and this report was limited to heterogeneous group of Army surgeons. Thus, the use of the MOTION platform to extract surgical details related to ACLR performed throughout the multiple MTFs may provide a more complete understanding of practice patterns among military surgeons, as these data can be collated across sites and used as a surrogate for a survey of surgical practices used to treat active duty members.

The purpose of this study is to evaluate current practice patterns in ACLR surgery among fellowshiptrained military surgeons. It was hypothesized that the bone-patellar tendon-bone (BPTB) autograft would be the most common graft choice and that independent femoral tunnel drilling would be the most commonly used technique among fellowship-trained military surgeons performing ACLR.

\section{Methods}

A retrospective review of prospectively collected intraoperative ACLR details performed between October 2016 and December 2019 by fellowshiptrained military orthopaedic surgeons was conducted. The MOTION database was queried using Current Procedural Terminology code 29888 for ACLR. Only active-duty members entered in the MOTION database between October 2016 and December 2019 involving either isolated primary ACLR and primary ACLR combined with meniscal or chondral injuries were included in the final analysis. All revision ACLRs, multiligamentous knee injuries (MLKIs), and primary ACLRs lacking documentation of graft fixation choice were excluded from analysis. Demographic information
Table 1. Patient Characteristics

\begin{tabular}{lr}
\hline & \multicolumn{1}{c}{ Value } \\
\hline Age, y (range) & $27.2 \pm 7.7(18-51)$ \\
Sex & $203(76.3)$ \\
$\quad$ Male & $63(23.7)$ \\
Female & \\
Active-duty status & $159(63.7)$ \\
$\quad$ Enlisted & $107(36.3)$ \\
$\quad$ Officer &
\end{tabular}

including patient age, sex, and rank were abstracted. The graft used was categorized as hamstring tendon (HT) autograft, BPTB, autograft, quadriceps tendon (QT) autograft, or allograft. Femoral tunnel drilling technique was categorized as either a transtibial or an independent technique. Femoral and tibial fixation methods were categorized as either interference screw or suspensory fixation. Concomitant meniscal pathology was reported as present or absent with specification of the site of pathology as either medial or lateral. Treatment of meniscal pathology was categorized as either debridement or repair. Meniscal repairs, when specified, were classified as all-inside repair, inside-out repair, outside-in repair, or meniscal transplantation. However, these variables were not uniformly reported among surgeons, which precluded analysis across the study group. In addition, the presence of concomitant chondral pathology and the type of chondral treatment were noted. Descriptive statistics were calculated using Microsoft Excel (Microsoft, Redmond, WA).

\section{Results}

Three hundred two primary ACLRs performed at 9 MTFs were identified in the initial screening of intraoperative details, and it was confirmed that all surgeons completed subspecialty fellowship training in arthroscopic knee surgery. All revision ACLRs (1) and 6 primary ACLR lacking documentation of graft fixation choice were excluded. Twenty-nine MLKI cases were excluded, leaving 266 primary ACLRs in the final analysis. The mean age of patient's undergoing ACLR was $27.2 \pm 7.7$ years. The majority of patients in this group were men, 203 of $266(76.3 \%)$ males versus 63 of $266(23.7 \%)$ females (Table 1). Isolated ACL tears were encountered in 99 of $266(37.2 \%)$ of total cases. ACL tears were encountered in conjunction with concomitant meniscal and/or chondral pathology in 168 of $266(63.2 \%)$ of cases.

Combined ACL and meniscal injuries were common (156 of 266 cases [58.6\%]), with comparable proportions of medial and lateral meniscal pathology noted (43.6\% medial, $34.6 \%$ lateral, $20.0 \%$ combined medial and lateral, $1.9 \%$ unspecified); $16.2 \%$ (43) of cases were combined with cartilage injury, and $11.7 \%$ (31) of 


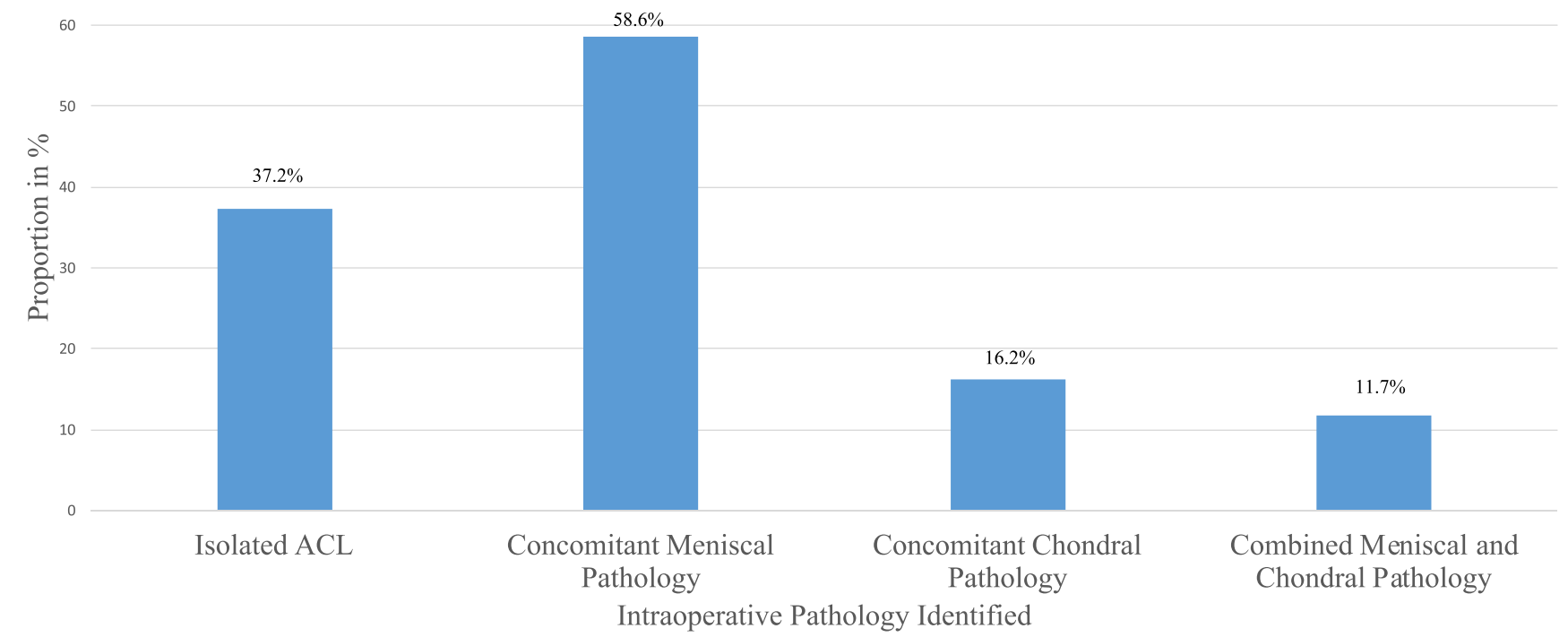

Fig 1. Rates of concomitant pathology identified intraoperatively. (ACL, anterior cruciate ligament.)

cases were combined with both meniscal and cartilage injury (Fig 1). Meniscal injuries were treated with an isolated debridement in 52 of $156(33.3 \%)$ tears, Meniscal repair was performed in 85 of $156(54.5 \%)$ tears. There were 8 of 156 cases $(5.1 \%)$ in which meniscal allograft transplantation was performed in conjunction with ACLR. In 11 of 156 cases $(7.1 \%)$ of meniscal tears, no treatment was reported (Table 2). Of the 85 reported meniscal repairs, an all-inside technique was used in 76 of 85 repairs $(89.4 \%)$, an insideout repair was performed in 8 of 85 repairs $(9.4 \%)$ of cases, and outside-in repair in 1 of $85(1.2 \%)$ repairs.

Concomitant chondral pathology was addressed with chondroplasty in 25 of $49(51.0 \%)$ lesions. In 14 of 49 cases $(28.6 \%)$, no treatment was performed. There was 1 case of concomitant osteochondral allograft transplant, 1 case treated with microfracture, and in 8 of 49 cases $(16.3 \%)$ of cases, treatment was not specifically recorded. The medial femoral condyle was the most common location for chondral defects, with 25 of 49 $(51.0 \%)$ followed by 7 of 49 (14.3\%) chondral lesions about the lateral femoral condyle. There were 7 of 49 $(14.3 \%)$ cases of patellar chondral defects, 6 of $49(12.2 \%)$ cases of trochlear chondral defects, and 4 of $49(8.2 \%)$ cases of tibial-sided osteochondral defects, and (Table 3).

BPTB autograft was the most commonly used graft source for ACLR, with 137 of 266 (51.5\%) cases, followed by HT autograft in 63 of $266(23.7 \%)$ of cases, and QT autograft in 42 of $266(15.8 \%)$ of cases. Allograft tissue was used for a graft source in 23 of $266(8.6 \%)$ of cases with an average age of 34.6 years (Fig 2). One of $266(0.3 \%)$ cases failed to specify graft choice. Femoral tunnel drill technique was recorded in $60.7 \%$ (160 of 266) of cases, all of which involved independent tunnel drilling. Of these 160 cases, an outside-in drilling technique or 2 -incision technique was used in $76.3 \%$ (122 of $160)$, and anteromedial portal technique was used in $23.8 \%$ (38 of 160). There were no cases in which the use of a transtibial technique for femoral tunnel drilling was recorded. A suspensory button was most commonly used for femoral-sided fixation (166 of 266 cases, $62.4 \%$ ), whereas an interference screw was used in 100 of $266(37.6 \%)$ cases. For tibial-sided fixation, an interference screw was used in a substantial majority of cases ( 210 of 266 cases, $78.9 \%$ ), whereas a suspensory button was used in 56 of $266(21.1 \%)$ cases.

\section{Discussion}

The most important findings of the current study are the majority of ACLRs performed by military surgeons involved independent femoral tunnel drilling, and BPTB autograft was the most commonly used graft choice. The majority $(54.5 \%)$ of meniscal tears were addressed with repair. This study represents the first of

Table 2. Meniscal Tear Location and Procedures

\begin{tabular}{lc}
\hline Meniscal tear location $(\mathrm{n}=156)$ & $68(43.6)$ \\
Medial & $54(34.6)$ \\
Lateral & $31(20.0)$ \\
Medial and Lateral & $3(1.9)$ \\
Unspecified & \\
Meniscal tear procedure & $52(33.3)$ \\
Debridement & $85(54.5)$ \\
Repair & $76(89.4)$ \\
All-inside & $8(9.4)$ \\
Inside-out & $1(1.2)$ \\
Outside-in & $8(5.1)$ \\
Allograft transplant & $11(7.1)$ \\
Unspecified & \\
\hline NOTE. Values are expressed as $\mathrm{n}(\%)$ unless otherwise noted
\end{tabular}


Table 3. Chondral Pathology Location and Procedures

\begin{tabular}{lr}
\hline Chondral pathology location $(\mathrm{n}=49)$ & \\
Medial femoral condyle & $25(51.0)$ \\
Lateral femoral condyle & $7(14.3)$ \\
Patella & $7(14.3)$ \\
Trochlea & $6(12.2)$ \\
Tibia & $4(8.2)$ \\
Chondral pathology procedure $(\mathrm{n}=49)$ & \\
No treatment & $14(28.6)$ \\
Chondroplasty & $25(51.0)$ \\
Marrow stimulation & $1(2.0)$ \\
Osteochondral allograft transplant & $1(2.0)$ \\
Unspecified & $8(16.4)$ \\
\hline
\end{tabular}

NOTE. Values are expressed as n (\%) unless otherwise noted.

its kind to employ the Department of Defense MOTION platform to characterize the surgical practice patterns of fellowship-trained military surgeons performing ACLR.

A number of studies have used surgeon surveys to characterize practice patterns related to ACLR in athletic populations, and these reports are important as they inform readers as to prevailing trends in surgical techniques and/or intraoperative decision-making. In 2002, Bradley et al. ${ }^{2}$ reported on the results of a survey distributed among 31 National Football League (NFL) team orthopaedic physicians, with $94 \%$ of those surveyed indicating a preference for patellar tendon autograft in the acute setting. Several other surveybased reports have demonstrated a similar preference of surgeons for BPTB autograft in ACLR among elite athletes. In 2014, Erickson et al. ${ }^{3}$ surveyed 267 NFL and National Collegiate Athletic Association team orthopaedic surgeons. Among the 137 respondents $(51 \%$ response rate) completing a 9-item survey, 118 of 137 $(86 \%)$ indicated their preference for BPTB autograft. Regarding femoral tunnel drilling technique, 90 of 137 $(66 \%)$ of surgeons reported using independent femoral tunnel drilling from an anteromedial portal, whereas $26 \%$ of respondents reported using a transtibial femoral tunnel drilling technique. ${ }^{3}$ In 2015, Erickson et al. ${ }^{4}$ published a subsequent report based on a similar survey of National Hockey League, Major League Soccer, and United States Olympic/World Cup Ski/Snowboard team orthopaedic surgeons. Similar to the survey of NFL and National Collegiate Athletic Association team orthopaedic surgeons completed the year prior, these authors noted a $50 \%$ response rate (47 of 94 orthopaedic surgeons surveyed.) Of these respondents, BPTB autograft was still used among a majority of team orthopaedic surgeons, albeit at a lower rate than previous reports (33 of 47 orthopaedic surgeons, $70 \%$ ). Interestingly, 21 of $47(44 \%)$ of respondents indicated a preference for drilling the femoral tunnel with a transtibial technique. ${ }^{4}$ There has been one recent survey of military orthopaedic surgeons pertaining to ACLR practice patterns. Synovec et al. ${ }^{5}$ queried 75 military surgeons who endorsed performing ACLR, 30 of whom $(40.5 \%)$ identified themselves as fellowshiptrained surgeons. The authors did not report specifically on the overall proportion of surgeons' graft preferences, instead stratifying graft preference as a function of patient age. Among male patients 25 years of age or younger, $51.3 \%$ of military surgeons indicated a preference for BPTB autograft, whereas among female patients 25 years of age or younger, only $34.2 \%$ of surgeons indicated a preference for BPTB autograft. Less than 3\% of military surgeons reported using QT autograft. In terms of femoral tunnel drilling technique and graft-fixation preference, the overwhelming majority $(96 \%)$ of military surgeons reported using an independent femoral tunnel drilling technique. Suspensory fixation for soft-tissue grafts was preferred by $87.7 \%$ of military surgeons, whereas interference screws were preferred for fixation of BPTB grafts by $88.7 \%$ of military surgeons. The current investigation demonstrates a similar preference for BPTB autograft and independent femoral tunnel among military surgeons as has been reported among surgeons treating elite athletes. However, relative to previously reported surveys, it would appear as though the popularity of QT autograft continues to grow.

The incidence of concomitant meniscal injuries encountered at the time of primary ACLR has been reported across multiple studies and has been found to be as high as $60 \% .{ }^{6,7}$ In a prospective analysis of 508 primary ACLRs analyzed by the MOON and MARS study groups, medial and lateral meniscal tears were noted in $40.3 \%$ and $45.8 \%$ of cases, respectively. ${ }^{7}$ Wyatt et al. ${ }^{8}$ performed a retrospective review of 261 patients who underwent primary ACLR and subsequent revision ACLR for recurrent instability. Among this group, meniscal pathology was encountered in 143 of $261(54.8 \%)$ of cases (85 medial meniscal tears [32.6\%] and 97 lateral meniscal tears [37.2\%]). Of the meniscal tears identified at the time of primary ACLR, either a medial or lateral meniscus repair was performed in only 35 of $261(13.4 \%)$ of cases. $^{8}$ In a separate series of 541 patients undergoing ACLR,
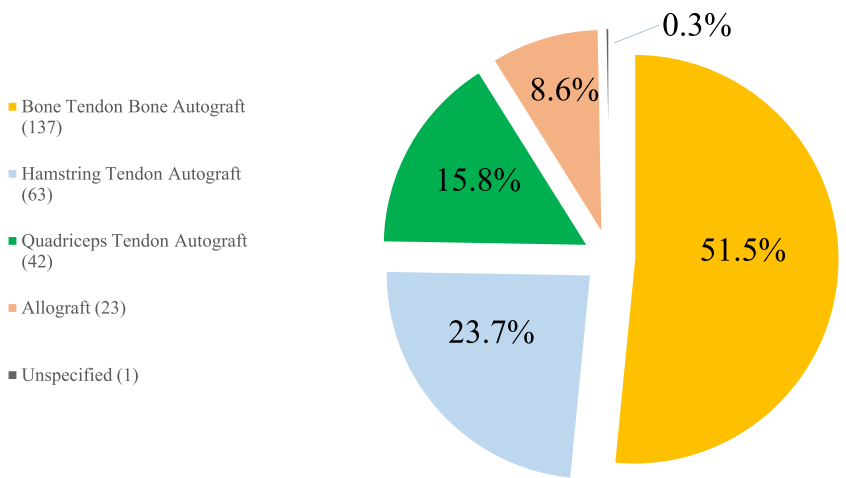

Fig 2. Graft choice. 
Table 4. Reported Rates of Meniscal Repairs Performed at the Time of ACL Reconstruction

\begin{tabular}{lcccc}
\hline \multicolumn{1}{c}{ Study } & Year Published & Group Size & Mean Age, y & Proportion Repaired, \% \\
\hline Noyes and Barber-Westin $^{10}$ & 2012 & 11711 & 29 & 26 \\
Kluczynski et al. $^{9}$ & 2013 & 408 & 25.9 & 19.8 \\
Wyatt et al. $^{8}$ & 2014 & 261 & 20 & 13.4 \\
Phillips et al. $^{11}$ & 2018 & 5391 & 27.2 & 27.2 \\
\hline
\end{tabular}

ACL, anterior cruciate ligament.

Kluczynski et al. ${ }^{9}$ observed meniscal tears in $75 \%$ of cases $(211$ of 408 [51.7\%] lateral meniscal tears, 197 of $408[48.3 \%]$ medial meniscal tears) and chondral injuries in $15.2 \%$ of cases. The majority of meniscal injuries in that series were treated with meniscectomy (205 of 408 [50.2\%] cases), whereas meniscal repair was performed in only 81 of $408(19.8 \%)$ of combined meniscal and ACL injuries. The rate of meniscectomy in that series was comparable to what Noyes and BarberWestin ${ }^{10}$ observed in a systematic review of 159 studies reporting on the treatment patterns of combined ACL and meniscal injuries. Among 11,711 meniscal tears, $7621(65 \%)$ were treated with meniscectomy, whereas only $3022(26 \%)$ of meniscal tears were repaired. ${ }^{10}$ More recently, Phillips et al. ${ }^{11}$ reported on the rate of meniscal repair performed at the time of primary ACLR using data derived from the Swedish National Knee Ligament Register (SNKLR). These authors noted that a medial meniscus repair was performed with ACLR in 588 of $2895(20.3 \%)$ cases and a lateral meniscus repair was performed in 323 of $2496(12.9 \%)$ cases. ${ }^{11}$ In the current study, the rate of concomitant meniscus pathology was similar to previous reports. However, the likelihood of meniscal repair performed at the time of primary ACLR among active-duty service members (85 of $156[54.5 \%]$ ]) was substantially greater than what has been previously been reported among comparably aged civilian groups (Table 4). ${ }^{8-11}$ This difference may be a representation of changing trends in practice, the unique demographics of the active-duty military population, and/or that military surgeons may be more aggressive in repairing meniscal tears identified at the time of ACLR when compared with rates of meniscal repairs performed by their civilian counterparts. However, it should be acknowledged that this is simply a qualitative observation, as the data do not allow for a quantitative comparison of meniscal repair rates between studies. That fact notwithstanding, the relatively high proportion of meniscal repairs performed by military surgeons may simply reflect a general trend toward increasing rates of meniscal repair among younger, more active patients. ${ }^{12,13}$ Across 9766 cases of combined ACLR and meniscal procedures performed by American Board of Orthopaedic Surgery Part II candidates, Cruz et al. ${ }^{14}$ noted greater rates of meniscal repairs performed by Sports Medicine and Pediatric orthopaedic fellowship-trained surgeons. A similar trend was noted by Wyatt et al., ${ }^{12}$ who observed that fellowship training was an independent predictor of meniscal repair performed in the setting of primary ACLR. It is therefore entirely possible the combination of factors related to the level of training of sampled surgeons and the unique demographic characteristics of this population may explain the preference towards meniscus repair in the current study.

With respect to the meniscal repair technique, military surgeons in this study overwhelmingly favored an all-inside repair technique, as $89.4 \%$ of meniscal tears were performed in this fashion. Although it is generally accepted that the popularity of the all-inside meniscus repair technique has grown in recent years, we are unaware of any literature pertaining to trends to suggest a preference among surgeons for one technique over another. However, a recent systematic review of 27 studies comparing all-inside with inside-out repairs found no differences in failure rates, functional outcomes, and complication rates. ${ }^{15}$

The incidence of chondral pathology noted at the time of ACLR has also been identified in multiple reports. ${ }^{6}$ In the aforementioned study published by the MOON and MARS study groups, Outerbridge grade 3 or 4 chondral lesions of the medial and lateral femoral condyles and patellofemoral joint were observed in $4.7 \%, 10.4 \%$, and $9.3 \%$ of cases. ${ }^{7}$ The incidence of concomitant chondral injuries in this series was similar, although the Outerbridge grade of those injuries was not commonly reported, limiting a more precise comparison with incidence rates in the published literature.

The MOON and MARS groups have provided working models for multicenter and multisurgeon initiatives to aggregate the pre-, intra-, and postoperative data pertaining to ACL injuries and associated pathology. Since its inception in 2002, MOON study group's multicenter/multisurgeon initiative has greatly enhanced contemporary understandings pertaining to the optimal graft choices for different patient populations, the incidence and effective treatments of concomitant meniscal and chondral pathology, effective rehabilitation guidelines postoperatively, and valuable longitudinal clinical outcomes data. ${ }^{16,17}$ It is our hope the MOTION platform will provide a similar apparatus for military surgeons working throughout multiple military MTFs to prospectively collect information to answer key clinical questions. 


\section{Limitations}

This study is not without limitations. We acknowledge that these data do not represent the totality of ACLR performed by fellowship-trained, military surgeons during the defined interval, as only those patients enrolled in MOTION would have intraoperative surveys completed by their surgeons. Moreover, the proportion of missing data, particularly as it pertains to femoral tunnel drilling method and outcome scores, limits a more complete interpretation of certain aspects of the practice patterns of this group of surgeons. However, the primary purpose of the current initiative was to determine the most commonly used graft type in ACLR. Presently, the number of patients enrolled in the MOTION varies from center to center and can be affected by access to dedicated research personnel and ancillary resources. At a number of participating centers, the activation of these resources has been incremental rather than instantaneous and universal. Consequently, the data in the current study are best viewed as a snapshot of current treatment practices rather than an exhaustive characterization of all ACLR performed during the defined interval. It should also be reiterated that these data only reflect the practice patterns of surgeons treating active-duty service members, and a number of ACLR performed on military health system beneficiaries were not included in the final analysis. In addition, this is a time zero study based on intraoperative data, which precludes any analysis of postoperative clinical outcomes. Moreover, information pertaining to perioperative practices such as return to full weight bearing and return to sport and return to duty rates are not available for analysis. Regarding graft type, HT grafts did not specify if they were 2- or 4 -stranded grafts, nor was information pertaining to sterilization technique of allograft tissue available for analysis.

\section{Conclusions}

BPTB autograft was the most commonly used graft type in ACLR among fellowship-trained surgeons treating active-duty service members. Concomitant meniscal pathology was encountered at rates comparable with what has been previously reported, and meniscal repair was favored over meniscal debridement in more than $50 \%$ cases.

\section{Acknowledgments}

The members of the MOTION Group are as follows: James R. Bailey, M.D., Travis C. Burns, M.D., Joseph R. Carney, M.D., Chad Haley, M.D., Lance E. LeClere, M.D., Timothy C. Mauntel, PhD., Lucas S. McDonald, M.D., Mark Pallis, D.O., Andrew N. Pike, M.D., Matthew A. Posner, M.D., Richard J. Robins, M.D., Ross A. Schumer, M.D., Mark A. Slabaugh, M.D., Jeffrey R.
Giuliani, M.D., Joseph T. Lanzi, M.D., Jennifer L. Smith, M.D., and William R. Volk, M.D.

The authors thank Daniel J. Cognetti, M.D., for his contributions to the preparation of the manuscript.

\section{References}

1. Ekhtiari S, Kay J, de Sa D, et al. What makes a successful survey? A systematic review of surveys used in anterior cruciate ligament reconstruction. Arthroscopy 2017;33: 1072-1079.e3.

2. Bradley JP, Klimkiewicz JJ, Rytel MJ, Powell JW. Anterior cruciate ligament injuries in the National Football League: Epidemiology and current treatment trends among team physicians. Arthroscopy 2002;18:502-509.

3. Erickson BJ, Harris JD, Fillingham YA, et al. Anterior cruciate ligament reconstruction practice patterns by NFL and NCAA football team physicians. Arthroscopy 2014;30: $731-738$.

4. Erickson BJ, Harris JD, Fillingham YA, et al. Orthopedic practice patterns relating to anterior cruciate ligament reconstruction in elite athletes. Am J Orthop (Belle Mead, NJ) 2015;44:E480-E485.

5. Synovec J, Shaw KA, Antosh IJ, et al. Current practices in anterior cruciate ligament reconstruction in the U.S. military: A survey of the society of military orthopaedic surgeons. Mil Med 2019;184:e249-e255.

6. Pike AN, Patzkowski JC, Bottoni CR. Meniscal and chondral pathology associated with anterior cruciate ligament injuries. J Am Acad Orthop Surg 2019;27:75-84.

7. Borchers JR, Kaeding CC, Pedroza AD, Huston LJ, Spindler KP, Wright RW. Intra-articular findings in primary and revision anterior cruciate ligament reconstruction surgery: A comparison of the MOON and MARS study groups. Am J Sports Med 2011;39:1889-1893.

8. Wyatt RW, Inacio MC, Liddle KD, Maletis GB. Prevalence and incidence of cartilage injuries and meniscus tears in patients who underwent both primary and revision anterior cruciate ligament reconstructions. Am J Sports Med 2014;42:1841-1846.

9. Kluczynski MA, Marzo JM, Bisson LJ. Factors associated with meniscal tears and chondral lesions in patients undergoing anterior cruciate ligament reconstruction: A prospective study. Am J Sports Med 2013;41:2759-2765.

10. Noyes FR, Barber-Westin SD. Treatment of meniscus tears during anterior cruciate ligament reconstruction. Arthroscopy 2012;28:123-130.

11. Phillips M, Ronnblad E, Lopez-Rengstig L, et al. Meniscus repair with simultaneous ACL reconstruction demonstrated similar clinical outcomes as isolated ACL repair: A result not seen with meniscus resection. Knee Surg Sports Traumatol Arthrosc 2018;26:2270-2277.

12. Wyatt RW, Inacio MC, Liddle KD, Maletis GB. Factors associated with meniscus repair in patients undergoing anterior cruciate ligament reconstruction. Am J Sports Med 2013;41:2766-2771.

13. Abrams GD, Frank RM, Gupta AK, Harris JD, McCormick FM, Cole BJ. Trends in meniscus repair and meniscectomy in the United States, 2005-2011. Am J Sports Med 2013;41:2333-2339. 
14. Cruz AI Jr, Gao B, Ganley TJ, et al. Trends in concomitant meniscal surgery among pediatric patients undergoing ACL reconstruction: An analysis of ABOS Part II Candidates From 2000 to 2016. Orthop J Sports Med 2019;7: 2325967119869848.

15. Fillingham YA, Riboh JC, Erickson BJ, Bach BR Jr, Yanke AB. Inside-out versus all-inside repair of isolated meniscal tears: An updated systematic review. Am J Sports Med 2017;45:234-242.
16. Krych AJ, Jackson JD, Hoskin TL, Dahm DL. A metaanalysis of patellar tendon autograft versus patellar tendon allograft in anterior cruciate ligament reconstruction. Arthroscopy 2008;24:292-298.

17. Lynch TS, Parker RD, Patel RM, et al. The Impact of the Multicenter Orthopaedic Outcomes Network (MOON) research on anterior cruciate ligament reconstruction and orthopaedic practice. J Am Acad Orthop Surg 2015;23: 154-163. 